\title{
Fixed point theorems for integral G-contractions
}

\author{
Maria Samreen ${ }^{1}$ and Tayyab Kamran ${ }^{2 *}$
}

${ }^{\text {*Correspondence: }}$

tayyabkamran@gmail.com

${ }^{2}$ Department of Mathematics,

Quaid-i-Azam University, Islamabad,

Pakistan

Full list of author information is

available at the end of the article

\begin{abstract}
We define the notion of an integral G-contraction for mappings on metric spaces and establish some fixed point theorems for such mappings. Our results generalize and unify some recent results by Jachymski, Branciari and those contained therein. As an application, we obtain a result for cyclic operators. Moreover, we provide an example to show that our results are substantial improvements of some known results in literature.
\end{abstract}

MSC: $47 \mathrm{H} 10 ; 54 \mathrm{H} 25$

Keywords: fixed points; directed graph; Picard operator

\section{Introduction}

Branciari [1] generalized the Banach contraction principle by proving the existence of a unique fixed point of a mapping on a complete metric space satisfying a general contractive condition of integral type. Afterwards, many authors undertook further investigations in this direction (see, e.g., [2-6]). Ran and Reurings [7] initiated the study of fixed points of mappings on partially ordered metric spaces. A number of interesting fixed point theorems have been obtained by different authors for this setting; see, for example, [7-11]. Jachymski [12] used the platform of graph theory instead of partial ordering and unified the results given by authors $[7,8,11]$. He showed that a mapping on a complete metric space still has a fixed point provided the mapping satisfies the contraction condition for pairs of points which form edges in the graph. Subsequently, Beg et al. [13] established a multivalued version of the main result of Jachymski [12]. Aydi et al. [14] studied fixed point theorems for weakly $G$-contraction mappings in $G$-metric spaces. Later on, Bojor [15] obtained some results in such settings by weakening the condition of Banach $G$-contractivity and introducing some new type of connectivity of a graph.

In this paper, motivated by the work of Jachymski [12] and Branciari [1], we introduce two new contraction conditions for mappings on complete metric spaces and, using these contractive conditions, obtain some fixed point theorems. Our results generalize and unify some results by the above mentioned authors.

\section{Preliminaries}

Let $(X, \preceq)$ be a partially ordered set. A mapping $f: X \rightarrow X$ is said to be nonincreasing if $x, y \in X, x \preceq y \Rightarrow f(x) \succeq f(y)$. A mapping $f$ is said to be nondecreasing if $x, y \in X, x \preceq y \Rightarrow$ $f(x) \preceq f(y)$. A mapping $f$ from a metric space $(X, d)$ into $(X, d)$ is called a Picard operator (PO) [11] if $f$ has a unique fixed point $t \in X$ and $\lim _{n \rightarrow \infty} f^{n} x=t$ for all $x \in X$. Two sequences 
$\left\{x_{n}\right\}$ and $\left\{y_{n}\right\}$ in a metric space $(X, d)$ are said to be equivalent if $d\left(x_{n}, y_{n}\right) \rightarrow 0$. Moreover, if each of them is Cauchy, then these are called Cauchy equivalent. A mapping from a metric space $(X, d)$ into $(X, d)$ is called orbitally continuous if for all $x, y \in X$ and any sequence $\left\{k_{n}\right\}$ of positive integers, $f^{k_{n}} x \rightarrow y$ implies $f\left(f^{k_{n}} x\right) \rightarrow f y$ as $n \rightarrow \infty$.

Let $G=(V(G), E(G))$ be a directed graph. By $G^{-1}$ we denote the graph obtained from $G$ by reversing the direction of edges, and by letter $\widetilde{G}$ we denote the undirected graph obtained from $G$ by ignoring the direction of edges. It will be more convenient to treat $\widetilde{G}$ as a directed graph for which the set of its edges is symmetric, i.e., $E(\widetilde{G})=E(G) \cup E\left(G^{-1}\right)$. If $x$ and $y$ are vertices in a graph $G$, then a path in $G$ from $x$ to $y$ of length $l$ is a sequence $\left(x_{i}\right)_{i=0}^{l}$ of $l+1$ vertices such that $x_{0}=x, x_{l}=y$ and $\left(x_{i-1}, x_{i}\right) \in E(G)$ for $i=1, \ldots, l$. A graph $G$ is called connected if there is a path between any two vertices. $G$ is weakly connected if $\widetilde{G}$ is connected. For a graph $G$ such that $E(G)$ is symmetric and $x$ is a vertex in $G$, the subgraph $G_{x}$ consisting of all edges and vertices which are contained in some path beginning at $x$ is called component of $G$ containing $x$. In this case $V\left(G_{x}\right)=[x]_{\widetilde{G}}$, where $[x]_{\tilde{G}}$ is the equivalence class of a relation $R$ defined on $V(G)$ by the rule: $y R z$ if there is a path in $G$ from $y$ to $z$. Clearly, $G_{x}$ is connected. A graph $G$ is known as a $(C)$-graph in $X$ [16] if for any sequence $\left\{x_{n}\right\}$ in $X$ with $x_{n} \rightarrow x$ and $\left(x_{n}, x_{n+1}\right) \in E(G)$ for $n \in \mathbb{N}$, there exists a subsequence $\left\{x_{n_{k}}\right\}$ of $\left\{x_{n}\right\}$ such that $\left(x_{n_{k}}, x\right) \in E(G)$ for $k \in \mathbb{N}$.

Subsequently, in this paper, $X$ is a complete metric space with metric $d$, and $\Delta$ is the diagonal of the Cartesian product $X \times X$. G is a directed graph such that the set $V(G)$ of its vertices coincides with $X$, and the set $E(G)$ of its edges contains all loops, i.e., $E(G) \supseteq \Delta$. Assume that $G$ has no parallel edges. We may treat $G$ as a weighted graph by assigning to each edge the distance between its vertices. A mapping $f: X \rightarrow X$ is called orbitally G-continuous [12] if for all $x, y \in X$ and any sequence $\left(k_{n}\right)_{n \in \mathbb{N}}$ of positive integers, $f^{k_{n}} x \rightarrow y$ and $\left(f^{k_{n}} x, f^{k_{n+1}} x\right) \in E(G), \forall n \in \mathbb{N}$ imply $f\left(f^{k_{n}} x\right) \rightarrow f y$.

We state, for convenience, the following definition and result.

Definition 2.1 [12, Definition 2.1] A mapping $f: X \rightarrow X$ is called a Banach G-contraction or simply a $G$-contraction if $f$ preserves edges of $G$, i.e.,

$$
\forall x, y \in X((x, y) \in E(G) \Rightarrow(f x, f y) \in E(G))
$$

and $f$ decreases weights of edges of $G$ in the following way:

$$
\exists c \in(0,1), \quad \forall x, y \in X((x, y) \in E(G) \Rightarrow d(f x, f y) \leq c d(x, y)) .
$$

Let $\Phi$ denote the class of all mappings $\phi:[0,+\infty) \rightarrow[0,+\infty)$ which are Lebesgue integrable, summable on each compact subset of $[0,+\infty)$, nonnegative and for each $\epsilon>0$, $\int_{0}^{\epsilon} \phi(s) d s>0$.

Theorem 2.2 [1, Theorem 2.1] Let $(X, d)$ be a complete metric space, $c \in(0,1)$, and let $f: X \rightarrow X$ be a mapping such that for each $x, y \in X$,

$$
\int_{0}^{d(f x, f y)} \phi(s) d s \leq c \int_{0}^{d(x, y)} \phi(s) d s
$$

where $\phi \in \Phi$. Then $f$ has a unique fixed point $t \in X$ such that for each $x \in X, \lim _{n \rightarrow \infty} f^{n} x=t$. 


\section{Main results}

We begin this section, motivated by Jachymski [12] and Branciari [1], by introducing the following definition.

Definition 3.1 A mapping $f: X \rightarrow X$ is called an integral G-contraction if $f$ preserves edges (see (2.1)) and

$$
\forall x, y \in X, \quad(x, y) \in E(G) \quad \text { implies } \quad \int_{0}^{d(f x, f y)} \phi(s) d s \leq c \int_{0}^{d(x, y)} \phi(s) d s
$$

for some $c \in(0,1)$ and $\phi \in \Phi$.

Remark 3.2 Note that if $f: X \rightarrow X$ satisfies (2.3), then $f$ is an integral $G_{1}$-contraction where $G_{1}=(X, X \times X)$. Moreover, every Banach $G$-contraction is an integral $G$-contraction (take $\phi(x)=1$ ), but the converse may not hold.

Proposition 3.3 Let $f: X \rightarrow X$ be an integral G-contraction with contraction constant $c \in(0,1)$ and $\phi \in \Phi$, then:

(i) $f$ is both an integral $G^{-1}$-contraction and an integral $\widetilde{G}$-contraction with the same contraction constant $c \in(0,1)$ and $\phi$.

(ii) $\left[x_{0}\right]_{\widetilde{G}}$ is $f$-invariant and $\left.f\right|_{\left[x_{0}\right]_{\tilde{G}}}$ is an integral $\widetilde{G}_{x_{0}}$-contraction provided that there exists some $x_{0} \in X$ such that $f x_{0} \in\left[x_{0}\right]_{\tilde{G}}$.

Proof (i) is a consequence of symmetry of $d$.

(ii) Let $x \in\left[x_{0}\right]_{\widetilde{G}}$. Then there is a path $x=z_{0}, z_{1}, \ldots, z_{m}=x_{0}$ between $x$ and $x_{0}$ in $\widetilde{G}$. Since $f$ is an integral $G$-contraction, then $\left(f z_{i-1}, f z_{i}\right) \in E(\widetilde{G}), \forall i=1,2, \ldots, l$. Thus $f x \in\left[f x_{0}\right]_{\widetilde{G}}=\left[x_{0}\right] \widetilde{G}$. Suppose that $(x, y) \in E\left(\widetilde{G}_{x_{0}}\right)$, then $(f x, f y) \in E(G)$ as $f$ is an integral G-contraction. But $\left[x_{0}\right]_{\widetilde{G}}$ is $f$ invariant, so we conclude that $(f x, f y) \in E\left(\widetilde{G}_{x_{0}}\right)$. Furthermore, (3.1) is satisfied automatically because $\widetilde{G}_{x_{0}}$ is a subgraph of $G$.

Lemma 3.4 Let $f: X \rightarrow X$ be an integral G-contraction and $y \in[x]_{\widetilde{G}}$, then

$$
\lim _{n \rightarrow \infty} d\left(f^{n} x, f^{n} y\right)=0
$$

Proof Let $x \in X$ and $y \in[x]_{\widetilde{G}}$, then there exists $N \in \mathbb{N}$ such that $x_{0}=x, x_{N}=y$ and $\left(x_{i-1}, x_{i}\right) \in$ $E(\widetilde{G})$ for all $i=1,2, \ldots, N$. By Proposition 3.3 it follows that $\left(f^{n} x_{i-1}, f^{n} x_{i}\right) \in E(\widetilde{G})$ and

$$
\int_{0}^{d\left(f^{n} x_{i-1}, f^{n} x_{i}\right)} \phi(s) d s \leq c \int_{0}^{d\left(f^{n-1} x_{i-1} f^{n-1} x_{i}\right)} \phi(s) d s
$$

holds for all $n \in \mathbb{N}$ and $i=0,1,2, \ldots, N$. Denote $d_{n}=d\left(f^{n} x_{i-1}, f^{n} x_{i}\right)$ for all $n \in \mathbb{N}$. If $d_{m}=0$ for some $m \in \mathbb{N}$, then it follows from (3.3) that $d_{n}=0$ for all $n \in \mathbb{N}$ with $n>m$. Therefore, in this case, $\lim _{n \rightarrow \infty} d_{n}=0$. Now, assume that $d_{n}>0$. We claim that $\left\{d_{n}\right\}$ is a non-increasing sequence. Otherwise, there exists $n_{\circ} \in \mathbb{N}$ such that $d_{n_{0}}>d_{n_{0}-1}$. Now, using the properties of $\phi$, it follows from (3.3) that

$$
0<\int_{0}^{d_{n_{0}-1}} \phi(s) d s \leq \int_{0}^{d_{n_{0}}} \phi(s) d s \leq c \int_{0}^{d_{n_{o}-1}} \phi(s) d s .
$$


Since, $0<c<1$, this yields a contradiction. Therefore, $\lim _{n \rightarrow \infty} d_{n}=r \geq 0$. Let $r>0$, then it follows from (3.3) that

$$
\begin{aligned}
\int_{0}^{r} \phi(s) d s & =\lim _{n \rightarrow \infty} \int_{0}^{d_{n}} \phi(s) d s \leq c \lim _{n \rightarrow \infty} \int_{0}^{d_{n-1}} \phi(s) d s \\
& =c \int_{0}^{r} \phi(s) d s,
\end{aligned}
$$

which implies $(1-c) \int_{0}^{r} \phi(s) d s \leq 0$ and it further implies that $\int_{0}^{r} \phi(s) d s=0$, a contradiction. Thus, $\lim _{n \rightarrow \infty} d_{n}=\lim _{n \rightarrow \infty} d\left(f^{n} x_{i-1}, f^{n} x_{i}\right)=0, \forall i=1,2, \ldots, N$, in both cases. From the triangular inequality, we have $d\left(f^{n} x, f^{n} y\right) \leq \sum_{i=1}^{N} d\left(f^{n} x_{i-1}, f^{n} x_{i}\right)$, and letting $n \rightarrow \infty$ gives $\lim _{n \rightarrow \infty} d\left(f^{n} x, f^{n} y\right)=0$.

Now we define a subclass of integral G-contractions. We call this a class of sub-integral $G$-contractions. Let us denote by $\Omega$ the class of all mappings $\phi \in \Phi$ satisfying following:

$$
\int_{0}^{\alpha+\beta} \phi(s) d s \leq \int_{0}^{\alpha} \phi(s) d s+\int_{0}^{\beta} \phi(s) d s
$$

for every $\alpha, \beta \geq 0$. Note that every constant function $\phi(x)=k>0$ belongs to the class $\Omega$.

Example 3.5 Define $\phi_{1}, \phi_{2}:[0, \infty) \rightarrow[0, \infty)$ by

$$
\phi_{1}(x)=\frac{1}{x+1}, \quad \phi_{2}(x)= \begin{cases}\frac{1}{2 \sqrt{x}}, & x \neq 0, \\ 0, & \text { otherwise }\end{cases}
$$

It is easy to see that $\phi_{1}, \phi_{2}$ satisfy (3.5) and thus belong to the class $\Omega$.

Definition 3.6 We say that an integral G-contraction is a sub-integral G-contraction if $\phi \in \Omega$.

Lemma 3.7 Let $f: X \rightarrow X$ be a sub-integral G-contraction and $y \in[x]_{\widetilde{G}}$. Then there exists $p(x, y) \geq 0$ such that

$$
\int_{0}^{d\left(f^{n} x, f^{n} y\right)} \phi(s) d s \leq c^{n} p(x, y), \quad \forall n \in \mathbb{N}
$$

Proof Let $x \in X$ and $y \in[x]_{\widetilde{G}}$, then there exists $N \in \mathbb{N}$ such that $x_{0}=x, x_{N}=y$ and $\left(x_{i-1}, x_{i}\right) \in$ $E(\widetilde{G})$ for all $i=1,2, \ldots, N$. Since $\phi \in \Omega$, using the triangular inequality, it follows that

$$
\int_{0}^{d\left(f^{n} x, f^{n} y\right)} \phi(s) d s \leq \sum_{i=1}^{N} \int_{0}^{d\left(f^{n} x_{i-1} f^{n} x_{i}\right)} \phi(s) d s .
$$

Moreover,

$$
\int_{0}^{d\left(f^{n} x_{i-1} f^{n} x_{i}\right)} \phi(s) d s \leq c^{n} \int_{0}^{d\left(x_{i-1}, x_{i}\right)} \phi(s) d s
$$


since $\left(f^{n} x_{i-1}, f^{n} x_{i}\right) \in E(G)$ for all $i=1,2,3, \ldots, N$ and $n \in \mathbb{N}$. From (3.7) and (3.8) we get

$$
\begin{aligned}
\int_{0}^{d\left(f^{n} x_{i} f^{n} y\right)} \phi(s) d s & \leq c^{n} \sum_{i=0}^{N} \int_{0}^{d\left(x_{i-1}, x_{i}\right)} \phi(s) d s \\
& =c^{n} p(x, y),
\end{aligned}
$$

where $p(x, y)=\sum_{i=0}^{N} \int_{0}^{d\left(x_{i-1}, x_{i}\right)} \phi(s) d s$.

Definition 3.8 Let $f: X \rightarrow X, y \in X$ and the sequence $\left\{f^{n} y\right\}$ in $X$ be such that $f^{n} y \rightarrow x^{*} \in X$ with $\left(f^{n} y, f^{n+1} y\right) \in E(G)$ for $n \in \mathbb{N}$. We say that the graph $G$ is a $\left(C_{f}\right)$-graph if there exists a subsequence $\left\{f^{n_{k}} y\right\}$ such that $\left(f^{n_{k}} y, x^{*}\right) \in E(G)$ for $k \in \mathbb{N}$.

Obviously, every $(C)$-graph is a $\left(C_{f}\right)$-graph for any self-mapping $f$ on $X$, but the converse may not hold as shown in the following.

Example 3.9 Let $X:=[0,1]$ with respect to the usual metric $d(x, y)=|x-y|$. Consider the graph $G$ consisting of $V(G):=X$ and $E(G):=\left\{\left(\frac{n}{n+1}, \frac{n+1}{n+2}\right): n \in \mathbb{N}\right\} \cup\left\{\left(\frac{x}{2^{n}}, \frac{x}{2^{n+1}}\right): n \in \mathbb{N}, x \in\right.$ $[0,1]\} \cup\left\{\left(\frac{x}{2^{2 n}}, 0\right): n \in \mathbb{N}, x \in[0,1]\right\}$. Note that $G$ is not a $(C)$-graph as $\frac{n}{n+1} \rightarrow 1$. Define $f:$ $X \rightarrow X$ as $f x=\frac{x}{2}$. Then $G$ is a $\left(C_{f}\right)$-graph since $f^{n} x=\frac{x}{2^{n}} \rightarrow 0$ for each $x \in[0,1]$.

Theorem 3.10 Let $f: X \rightarrow X$ be a sub-integral G-contraction. Assume that

(i) $X_{f}:=\{x \in X:(x, f x) \in E(G)\} \neq \emptyset$,

(ii) $G$ is a $\left(C_{f}\right)$-graph.

Then, for any $z_{0} \in X_{f},\left.f\right|_{\left[z_{0}\right]_{\widetilde{G}}}$ is a Picard operator. Further, if $G$ is weakly connected, then $f$ is a Picard operator.

Proof Let $z_{0} \in X_{f}$, then $f z_{0} \in\left[z_{0}\right]_{\widetilde{G}}$. Let $m>n \geq 1$, using Lemma 3.7, we have

$$
\begin{aligned}
\int_{0}^{d\left(f^{m} z_{0} f^{n} z_{0}\right)} \phi(s) d s & \leq \int_{0}^{d\left(f^{n} z_{0}, f^{n+1} z_{0}\right)} \phi(s) d s+\cdots+\int_{0}^{d\left(f^{m-1} z_{0}, f^{m} z_{0}\right)} \phi(s) d s \\
& \leq\left(c^{n}+c^{n+1}+c^{n+2}+\cdots+c^{m-1}\right) p\left(z_{0}, f z_{0}\right) \\
& \leq\left[\frac{c^{n}}{1-c}\right] p\left(z_{0}, f z_{0}\right) \rightarrow 0 \quad \text { as } n \rightarrow \infty .
\end{aligned}
$$

It follows that $\left\{f^{n} z_{0}\right\}$ is a Cauchy sequence in $X$. Therefore, $f^{n} z_{0} \rightarrow t \in X$. Let $y$ be another element in $\left[z_{0}\right]_{\widetilde{G}}$, then it follows from Lemma 3.4 that $f^{n} y \rightarrow t$, too. Next, we show that $t$ is a fixed point of $f$. Since $f^{n} z_{0} \rightarrow t \in X$ and $\left(f^{n} z_{0}, f^{n+1} z_{0}\right) \in E(G)$ for all $n \in \mathbb{N}$ and $G$ is a $\left(C_{f}\right)$-graph, then there exists a subsequence $\left\{f^{n_{k}} z_{0}\right\}$ of $\left\{f^{n} z_{0}\right\}$ such that $\left(f^{n_{k}} z_{0}, t\right) \in E(G)$ for all $k \in \mathbb{N}$. Therefore, $\left(z_{0}, f z_{0}, f^{2} z_{0}, \ldots, f^{n_{1}} z_{0}, t\right)$ is a path in $G$ and so in $\widetilde{G}$ from $z_{0}$ to $t$, thus $t \in\left[z_{0}\right]_{\widetilde{G}}$. From (3.1), we get

$$
\int_{0}^{d\left(f^{n_{k}+1} z_{0}, f t\right)} \phi(s) d s \leq c \int_{0}^{d\left(f^{n_{k}} z_{0}, t\right)} \phi(s) d s, \quad \forall k \in \mathbb{N},
$$

letting $k \rightarrow \infty$, we have $\int_{0}^{d(t, f t)} \phi(s) d s=0$, which implies that $d(t, f t)=0$. This shows that $\left.f\right|_{\left[z_{0}\right]_{\tilde{G}}}$ is a Picard operator. Moreover, if $G$ is weakly connected, then $f$ is a Picard operator since $\left[z_{0}\right]_{\tilde{G}}=X$. 
Corollary 3.11 Let $(X, d)$ be a complete metric space endowed with a graph $G$ such that $G$ is $\left(C_{f}\right)$-graph. Then the following statements are equivalent:

(1) $G$ is weakly connected.

(2) Every sub-integral G-contraction $f$ on $X$ is a Picard operator provided that $X_{f} \neq \emptyset$.

Proof $(1) \Rightarrow(2)$ : It is immediate from Theorem 3.10.

$(2) \Rightarrow(1)$ : On the contrary, suppose that $G$ is not weakly connected, then $\widetilde{G}$ is disconnected, i.e., there exists $x_{\circ} \in X$ such that $\left[x_{\circ}\right]_{\widetilde{G}} \neq \emptyset$ and $X \backslash\left[x_{\circ}\right]_{\tilde{G}} \neq \emptyset$. Let $y_{\circ} \in X \backslash\left[x_{\circ}\right]_{\widetilde{G}}$, we construct a self-mapping $f$ by (as in [12, Theorem 3.1]):

$$
f x= \begin{cases}x_{\circ} & \text { if } x \in\left[x_{\circ}\right]_{\widetilde{G}}, \\ y_{\circ} & \text { if } x \in X \backslash\left[x_{\circ}\right]_{\widetilde{G}} .\end{cases}
$$

Let $(x, y) \in E(G)$, then $[x]_{\widetilde{G}}:=[y]_{\widetilde{G}}$, which implies $f x=f y$ hence $(f x, f y) \in E(G)$, since $G$ contains all loops and further (3.1) is trivially satisfied (take $\phi(s)=1$ ). But $x_{\circ}$ and $y_{\circ}$ are two fixed points of $f$ contradicting the fact that $f$ has a unique fixed point.

Theorem 3.12 Let $f: X \rightarrow X$ be a sub-integral G-contraction. Assume that $f$ is orbitally G-continuous and $X_{f}:=\{x \in X:(x, f x) \in E(G)\} \neq \emptyset$. Then, for any $z_{0} \in X_{f}$ and $y \in\left[z_{0}\right]_{\widetilde{G}}$, $\lim _{n \rightarrow \infty} f^{n} y=t \in X$ where $t$ is a fixed point of $f$. Further, if $G$ is weakly connected, then $f$ is a Picard operator.

Proof Let $z_{0} \in X_{f}$, then the arguments used in the proof of Theorem 3.10 imply that $\left\{f^{n} z_{0}\right\}$ is a Cauchy sequence. Therefore, $f^{n} z_{0} \rightarrow t \in X$. Since $\left(f^{n} z_{0}, f^{n+1} z_{0}\right) \in E(G)$ for all $n \in \mathbb{N}$ and $f$ is orbitally $G$-continuous, therefore $t=\lim _{n \rightarrow \infty} f f^{n} z_{0}=f t$. Note that if $y$ is another element from $\left[z_{0}\right]_{\widetilde{G}}$, then it follows from Lemma 3.4 that $\lim _{n \rightarrow \infty} f^{n} y=t$. Finally, if $G$ is weakly connected, then $\left[z_{0}\right]_{\widetilde{G}}:=X$, which yields that $f$ is a Picard operator.

Remark 3.13 Theorem 3.12 generalizes claims $2^{0} \& 3^{0}$ of [12, Theorem 3.3].

Theorem 3.14 Let $f: X \rightarrow X$ be a sub-integral G-contraction. Assume that $f$ is orbitally continuous and if there exists some $z_{0} \in X$ such that $f z_{0} \in\left[z_{0}\right]_{\widetilde{G}}$, then, for $y \in\left[z_{0}\right]_{\widetilde{G}}$, $\lim _{n \rightarrow \infty} f^{n} y=t \in X$, where $t$ is a fixed point of $f$. Further, if $G$ is weakly connected, then $f$ is a Picard operator.

Proof Let $z_{0} \in X$ be such that $f z_{0} \in\left[z_{0}\right]_{\widetilde{G}}$, then using the same arguments as in the proof of Theorem 3.10, $\left\{f^{n} z_{0}\right\}$ is Cauchy and thus $\lim _{n \rightarrow \infty} f^{n} z_{0}=t \in X$. Moreover, $t=$ $\lim _{n \rightarrow \infty} f^{n} z_{0}=f t$, since $f$ is orbitally continuous. Note that if $y$ is another element from $\left[z_{0}\right]_{\widetilde{G}}$, then it follows from Lemma 3.4 that $\lim _{n \rightarrow \infty} f^{n} y=t$. If $G$ is weakly connected, then $\left[z_{0}\right]_{\widetilde{G}}:=X$. This yields that $f$ is a Picard operator.

Remark 3.15 Theorem 3.14 generalizes claims $2^{0} \& 3^{0}$ of [12, Theorem 3.4] and thus generalizes and extends the results of Nieto and Rodrýguez-López [8, Theorems 2.1 and 2.3], Petrusel and Rus [11, Theorem 4.3] and Ran and Reurings [7, Theorem 2.1].

Corollary 3.16 Let $(X, d)$ be a complete metric space endowed with a graph $G$. Then the following statements are equivalent: 
(1) G is weakly connected.

(2) Every sub-integral G-contraction $f$ on $X$ is a Picard operator provided that $f$ is orbitally continuous.

Proof $(1) \Rightarrow(2)$ is obvious from Theorem 3.14. Note that the example constructed in Corollary 3.11 is orbitally continuous. Hence, $(2) \Rightarrow(1)$.

Remark 3.17 Corollary 3.16 generalizes claims $2^{0} \& 3^{0}$ of [12, Corollary 3.3].

Kirk et al. [17] introduced cyclic representations and cyclic contractions and they have been further investigated by many authors (see, e.g., [18-20]). Let $X$ be a nonempty set, $m$ be a positive integer and $\left\{A_{i}\right\}_{i=1}^{m}$ be nonempty closed subsets of $X$ and $f: \bigcup_{i=1}^{m} A_{i} \rightarrow \bigcup_{i=1}^{m} A_{i}$ be an operator. Then $X:=\bigcup_{i=1}^{m} A_{i}$ is known as a cyclic representation of $X$ w.r.t. $f$ if

$$
f\left(A_{1}\right) \subset A_{2}, \ldots, \quad f\left(A_{m-1}\right) \subset A_{m}, \quad f\left(A_{m}\right) \subset A_{1}
$$

and the operator $f$ is known as a cyclic operator.

Theorem 3.18 Let $(X, d)$ be a complete metric space. Let $m$ be a positive integer, $\left\{A_{i}\right\}_{i=1}^{m}$ be nonempty closed subsets of $X, Y:=\bigcup_{i=1}^{m} A_{i}$ and $f: Y \rightarrow Y$. Assume that

(i) $\bigcup_{i=1}^{m} A_{i}$ is a cyclic representation of $Y$ w.r.t.f;

(ii) there exists $\phi \in \Omega$ such that $\int_{0}^{d\left(f x_{x} f y\right)} \phi(s) d s \leq c \int_{0}^{(d(x, y))} \phi(s) d s$ whenever, $x \in A_{i}$, $y \in A_{i+1}$, where $A_{m+1}=A_{1}$.

Then $f$ has a unique fixed point $t \in \bigcap_{i=1}^{m} A_{i}$ and $f^{n} y \rightarrow t$ for any $y \in \bigcup_{i=1}^{m} A_{i}$.

Proof We note that $(Y, d)$ is a complete metric space. Let us consider a graph $G$ consisting of $V(G):=Y$ and $E(G):=\Delta \cup\left\{(x, y) \in Y \times Y: x \in A_{i}, y \in A_{i+1} ; i=1, \ldots, m\right\}$. By (i) and (ii) it follows that $f$ is a sub-integral G-contraction. Now let $f^{n} x \rightarrow x^{n}$ in $Y$ such that $\left(f^{n} x, f^{n+1} x\right) \in$ $E(G)$ for all $n \geq 1$. Then in view of (3.10), the sequence $\left\{f^{n} x\right\}$ has infinitely many terms in each $A_{i}$ so that one can easily extract a subsequence of $\left\{f^{n} x\right\}$ converging to $x^{\prime \prime}$ in each $A_{i}$. Since $A_{i}$ 's are closed, then $x^{*} \in \bigcap_{i=1}^{m} A_{i}$. Now it is easy to form a subsequence $\left\{f^{n_{k}} x\right\}$ in some $A_{j}, j \in\{1, \ldots, m\}$ such that $\left(f^{n_{k}} x, x^{*}\right) \in E(G)$ for $k \geq 1$, it vindicates $G$ is a weakly connected $\left(C_{f}\right)$-graph and thus conclusion follows from Theorem 3.10.

Remark 3.19 Taking $\phi(s)=1$, Theorem 3.18 subsumes the main result of [17].

Theorem 3.20 Let $f: X \rightarrow X$ be an integral G-contraction. Assume that the following assertions hold:

(i) there exists $z_{0} \in X_{f}$ such that

$$
\int_{0}^{d(f x, f y)} \phi(s) d s \leq c \int_{0}^{d(x, y)} \phi(s) d s, \quad \forall x, y \in \mathcal{O}\left(z_{0}\right) \subset\left[z_{0}\right]_{\widetilde{G}},
$$

where $\mathcal{O}\left(z_{0}\right)=\left\{z_{0}, f z_{0}, f^{2} z_{0}, \ldots\right\}$.

(ii) $G$ is a $\left(C_{f}\right)$-graph.

Then, for any $z_{0} \in X_{f},\left.f\right|_{\left[z_{0}\right] \tilde{G}}$ is a Picard operator. Furthermore, if $G$ is weakly connected then $f$ is a Picard operator. 
Proof Let $z_{0} \in X_{f}$, then $f z_{0} \in\left[z_{0}\right]_{\widetilde{G}}$. Now, it follows from Proposition 3.3(ii) that $\mathcal{O}\left(z_{0}\right) \subset$ $\left[z_{0}\right]_{\widetilde{G}}$. Moreover, from Lemma 3.4 we get

$$
\lim _{n \rightarrow \infty} d\left(f^{n} z_{0}, f^{n+1} z_{0}\right)=0
$$

We claim that $\left\{f^{n} z_{0}\right\}$ is Cauchy sequence. Otherwise, there exists some $\epsilon>0$ in such a way that, for each $k \in \mathbb{N}$, there are $m_{k}, n_{k} \in \mathbb{N}$ with $n_{k}>m_{k}>k$ satisfying

$$
d\left(f^{m_{k}} z_{0}, f^{n_{k}} z_{0}\right) \geq \epsilon .
$$

We may choose sequences $\left\{m_{k}\right\},\left\{n_{k}\right\}$ such that corresponding to $m_{k}$, natural number $n_{k}$ is smallest satisfying (3.13). Therefore,

$$
\epsilon \leq d\left(f^{n_{k}} z_{0}, f^{m_{k}} z_{0}\right) \leq d\left(f^{n_{k}} z_{0}, f^{n_{k}-1} z_{0}\right)+d\left(f^{n_{k}-1} z_{0}, f^{m_{k}} z_{0}\right)<d\left(f^{n_{k}} z_{0}, f^{n_{k}-1} z_{0}\right)+\epsilon .
$$

On letting $k \rightarrow \infty$ and using (3.12), we get

$$
\lim _{k \rightarrow \infty} d\left(f^{n_{k}} z_{0}, f^{m_{k}} z_{0}\right)=\epsilon
$$

Moreover, using (3.12) and (3.14), it follows from

$$
d\left(f^{n_{k}-1} z_{0}, f^{m_{k}-1} z_{0}\right) \leq d\left(f^{n_{k}-1} z_{0}, f^{n_{k}} z_{0}\right)+d\left(f^{n_{k}} z_{0}, f^{m_{k}} z_{0}\right)+d\left(f^{m_{k}} z_{0}, f^{m_{k}-1} z_{0}\right)
$$

and

$$
d\left(f^{n_{k}} z_{0}, f^{m_{k}} z_{0}\right) \leq d\left(f^{n_{k}} z_{0}, f^{n_{k}-1} z_{0}\right)+d\left(f^{n_{k}-1} z_{0}, f^{m_{k}-1} z_{0}\right)+d\left(f^{m_{k}-1} z_{0}, f^{m_{k}} z_{0}\right)
$$

that

$$
\lim _{n \rightarrow \infty} d\left(f^{n_{k}-1} z_{0}, f^{m_{k}-1} z_{0}\right)=\epsilon
$$

Since $f^{n_{k}-1} z_{0}, f^{m_{k}-1} z_{0} \in \mathcal{O}\left(z_{0}\right)$, it follows from assertion (ii) that

$$
\int_{0}^{d\left(f^{n} z_{0}, f^{m} z_{0}\right)} \phi(s) d s \leq c \int_{0}^{d\left(f^{n} k^{-1} z_{0} f^{m_{k}-1} z_{0}\right)} \phi(s) d s .
$$

Letting $k \rightarrow \infty$ and using (3.14), (3.15), we get $(1-c) \int_{0}^{\epsilon} \phi(s) d s \leq 0$. As $0<c<1$, this implies that $\epsilon=0$. Therefore, $\left\{f^{n} z_{0}\right\}$ is a Cauchy sequence in $X$. The rest of the proof runs on the same lines as the proof of Theorem 3.10.

Remark 3.21 Theorem 3.20 generalizes [1, Theorem 2.1].

Remark 3.22 The conclusion of Theorem 3.20 that $f$ is a Picard operator remains valid if we replace assertion (ii) by (ii)' $f$ is orbital G-continuous or (ii)" $f$ is orbitally continuous. 
Example 3.23 Let $X:=[0,1]$ be equipped with the usual metric $d$. Define $f: X \rightarrow X$, $\phi:[0,+\infty) \rightarrow[0,+\infty)$ by

$$
f x=\left\{\begin{array}{ll}
\frac{x}{1+p x} & \text { if } x=\frac{1}{n}, \\
0 & \text { if } x \neq \frac{1}{n}
\end{array} \text { and } \quad \phi(s)= \begin{cases}s^{\frac{1}{s}-2}(1-\log s) & \text { if } s>0 \\
0 & \text { if } s=0\end{cases}\right.
$$

for all $n \in \mathbb{N}$ and $p \geq 1$ is any fixed positive integer. Consider the graph $G$ such that $V(G):=$ $X$ and $E(G):=\Delta \cup\{(0, x): x \in X\} \cup\left\{\left(\frac{1}{n+1}, \frac{1}{n}\right): n \in \mathbb{N}\right\}$. We observe that (2.1) holds. Moreover, $\int_{0}^{\tau} \phi(s) d s=\tau^{\frac{1}{\tau}}$, so that (3.1) is equivalent to

$$
d(f x, f y)^{\frac{1}{d(x, f y)}} \leq c d(x, y)^{\frac{1}{d(x, y)}} \quad \text { for }(x, y) \in E(G) .
$$

Next we show that (3.17) is satisfied for $c=\frac{1}{1+p}<1$.

Case i. Let $(x, x) \in E(G)$, then (3.1) is trivially satisfied.

Case ii. Let $(0, x) \in E(G) ; x \neq \frac{1}{n}$ for $n \in \mathbb{N},(3.1)$ is trivially satisfied. Let $(0, x) \in E(G) ; x=\frac{1}{n}$ for $n \in \mathbb{N}$

$$
d(f x, f y)^{\frac{1}{d(x x, f y)}}=\left|\frac{1}{n+p}-0\right|^{\frac{1}{\frac{1}{n+p}-0 \mid}}=\frac{1}{(n+p)^{(n+p)}}
$$

and

$$
d(x, y)^{\frac{1}{d(x, y)}}=\left|\frac{1}{n}-0\right|^{\mid \frac{1}{\left|\frac{1}{n}-0\right|}}=\frac{1}{(n)^{n}} .
$$

From inequality (3.17) we need to show that

$$
\frac{1}{(n+p)^{(n+p)}} \leq \frac{1}{(1+p)(n)^{n}}
$$

or, equivalently,

$$
\left[\frac{n}{n+p}\right]^{n} \frac{1}{(n+p)^{p}} \leq \frac{1}{1+p} .
$$

Since $\frac{1}{(n+p)^{p}} \leq \frac{1}{(n+p)}<\frac{1}{(1+p)}$ for all $n \in \mathbb{N}$ and $\frac{n}{n+p}<1$, thus inequality (3.20) is satisfied.

Case iii. Let $\left(\frac{1}{n+1}, \frac{1}{n}\right) \in E(G)$ for $n \in \mathbb{N}$, then we have

$$
d(f x, f y)^{\frac{1}{d(x, f y)}}=\left|\frac{1}{n+1+p}-\frac{1}{n+p}\right|^{\frac{1}{\frac{1}{n+1+p}-\frac{1}{n+p} \mid}}=\left[\frac{1}{(n+1+p)(n+p)}\right]^{(n+1+p)(n+p)}
$$

and

$$
d(x, y)^{\frac{1}{d(x, y)}}=\left|\frac{1}{n}-\frac{1}{n+1}\right|^{\frac{1}{\left|\frac{1}{n}-\frac{1}{n+1}\right|}}=\left[\frac{1}{n(n+1)}\right]^{n(n+1)},
$$

so we need to show that

$$
\left[\frac{1}{(n+1+p)(n+p)}\right]^{(n+1+p)(n+p)} \leq \frac{1}{(1+p)}\left[\frac{1}{n(n+1)}\right]^{n(n+1)} .
$$


On rearranging we have

$$
\frac{[n(n+1)]^{n(n+1)}}{[(n+1+p)(n+p)]^{(n+1+p)(n+p)}} \leq \frac{1}{(p+1)}
$$

or

$$
\left[\frac{n}{n+p}\right]^{n(n+1)}\left[\frac{n+1}{n+1+p}\right]^{n(n+1)} \frac{1}{[(n+1+p)(n+p)]^{\left(p^{2}+2 n p+p\right)}} \leq \frac{1}{1+p}
$$

By analyzing L.H.S., we see that $\frac{n}{n+p}<1$ and $\frac{n+1}{n+1+p}<1$ for all $n \in \mathbb{N}$ and $\frac{1}{[(n+1+p)(n+p)]^{\left(p^{2}+2 n p+p\right)}}<$ $\frac{1}{[(n+1+p)(n+p)]}<\frac{1}{(n+p)} \leq \frac{1}{(1+p)}$ for all $n \in \mathbb{N}$, which infers that inequality (3.23) is indeed true. Therefore, $f$ is an integral $G$-contraction with contraction constant $c=\frac{1}{1+p}$. Note that $G$ is a weakly connected $(C)$-graph and (3.11) also holds for $\mathcal{O}(0)$. Thus all the conditions of Theorem 3.20 are satisfied and $f$ is a Picard operator with fixed point 0 . Note that $f$ is not a Banach $G$-contraction since, for $\left(\frac{1}{n+1}, \frac{1}{n}\right) \in E(G)$,

$$
\frac{d\left(f \frac{1}{n+1}, f \frac{1}{n}\right)}{d\left(\frac{1}{n+1}, \frac{1}{n}\right)}=\frac{\left|\frac{1}{n+1+p}-\frac{1}{n+p}\right|}{\left|\frac{1}{n}-\frac{1}{n+1}\right|} \rightarrow 1 \quad \text { as } n \rightarrow \infty .
$$

By setting $p=2$ in the above example, we have

$$
d\left(f \frac{1}{2}, f \frac{3}{4}\right)^{\frac{1}{d\left(\frac{1}{2}, f \frac{3}{4}\right)}}=\left(\frac{1}{4}\right)^{4}=d\left(\frac{1}{2}, \frac{3}{4}\right)^{\frac{1}{d\left(\frac{1}{2}, \frac{3}{4}\right)}} .
$$

Therefore one cannot apply Theorem 2.2 [1].

\section{Conclusion}

The notion of an integral G-contraction not only generalizes/extends the notion of a Banach G-contraction, but it also improves the integral inequality (2.3). Whereas, the notion of a sub-integral $G$-contraction generalizes the notion of a Banach $G$-contraction, but it partially generalizes the integral inequality (2.3). Therefore, Theorem 3.10 generalizes/extends some results of Jachymski [12] and provides partial improvement to the main result of Branciari [1]. A very natural question is bound to be posed: Are the conclusions of Theorems 3.10, 3.12, 3.14 still valid for integral G-contractions? In Theorem 3.20, we have provided a partial answer to this question by imposing condition (3.11). But it remains open to investigate an affirmative answer without the crucial condition of (3.11). Furthermore, Example 3.23 invokes the generality of Theorem 3.20.

Competing interests

The authors declare that they have no competing interests.

Authors' contributions

Both authors contributed equally in this article.

\section{Author details}

${ }^{1}$ Centre for Advanced Mathematics and Physics, National University of Sciences and Technology, H-12, Islamabad,

Pakistan. ${ }^{2}$ Department of Mathematics, Quaid-i-Azam University, Islamabad, Pakistan.

\section{Acknowledgements}

Authors are grateful to referees for their suggestions. 


\section{References}

1. Branciari, A: A fixed point theorem for mappings satisfying a general contractive condition of integral type. Int. J. Math. Math. Sci. 29, 531-536 (2002)

2. Aliouche, A: A common fixed point theorem for weakly compatible mappings in symmetric spaces satisfying a contractive condition of integral type. J. Math. Anal. Appl. 322, 796-802 (2006)

3. Djoudi, A, Aliouche, A: Common fixed point theorems of Gregus type for weakly compatible mappings satisfying contractive conditions of integral type. J. Math. Anal. Appl. 329, 31-45 (2007)

4. Rhoades, BE: Two fixed-point theorems for mappings satisfying a general contractive condition of integral type. Int. J. Math. Math. Sci. 63, 4007-4013 (2003)

5. Suzuki, T: Meir-Keeler contractions of integral type are still Meir-Keeler contractions. Int. J. Math. Math. Sci. 2007, Article ID 39281 (2007)

6. Vijayaraju, P, Rhoades, BE, Mohanraj, R: A fixed point theorem for a pair of maps satisfying a general contractive condition of integral type. Int. J. Math. Math. Sci. 15, 2359-2364 (2005)

7. Ran, ACM, Reurings, MCB: A fixed point theorem in partially ordered sets and some applications to matrix equations. Proc. Am. Math. Soc. 132, 1435-1443 (2004)

8. Nieto, JJ, Rodríguez-López, R: Contractive mapping theorems in partially ordered sets and applications to ordinary differential equations. Order 22, 223-239 (2005)

9. Nieto, JJ, Pouso, RL, Rodríguez-López, R: Fixed point theorems in ordered abstract spaces. Proc. Am. Math. Soc. 135, 2505-2517 (2007)

10. Pathak, HK, Tiwari, S: Common fixed point and best simultaneous approximations for Ciric type $(f, g)$-weak contraction and weak asymptotic contraction. Int. J. Pure Appl. Math. 62, 291-304 (2010)

11. Petrusel, A, Rus, IA: Fixed point theorems in ordered L-spaces. Proc. Am. Math. Soc. 134, 411-418 (2006)

12. Jachymski, J: The contraction principle for mappings on a metric space with a graph. Proc. Am. Math. Soc. 136, 1359-1373 (2007)

13. Beg, I, Butt, AR, Radojević, S: The contraction principle for set valued mappings on a metric space with a graph Comput. Math. Appl. 60, 1214-1219 (2010)

14. Aydi, H, Shatanawi, W, Vetro, C: On generalized weakly G-contraction mapping in G-metric spaces. Comput. Math. Appl. 62, 4222-4229 (2011)

15. Bojor, F: Fixed point theorems for Reich type contractions on metric spaces with a graph. Nonlinear Anal. 75, 3895-3901 (2012)

16. Aleomraninejad, SMA, Rezapour, S, Shahzad, N: Some fixed point results on a metric space with a graph. Topol. Appl. $159,659-663$ (2012)

17. Kirk, WA, Srinivasan, PS, Veeranmani, P: Fixed points for mappings satisfying cyclical contractive condition. Fixed Point Theory 4(1), 79-89 (2003)

18. Karapinar, E, Sadarangani, K: Fixed point theory for cyclic $(\varphi-\psi)$-contractions. Fixed Point Theory Appl. 2011, 69 (2011)

19. Alghamdi, MA, Petrusel, A, Shahzad, N: A fixed point theorem for cyclic generalized contractions in metric spaces. Fixed Point Theory Appl. 2012, 122 (2012)

20. Petric, MA: Some remarks concerning Ċiric̀-Reich-Rus operators. Creat. Math. Inf. 18, 188-193 (2009)

doi:10.1186/1687-1812-2013-149

Cite this article as: Samreen and Kamran: Fixed point theorems for integral G-contractions. Fixed Point Theory and Applications 2013 2013:149.

\section{Submit your manuscript to a SpringerOpen ${ }^{\circ}$ journal and benefit from:}

- Convenient online submission

- Rigorous peer review

- Immediate publication on acceptance

- Open access: articles freely available online

- High visibility within the field

- Retaining the copyright to your article 